\title{
DNA Polymerase Profiling
}

\section{Daniel Summerer}

\begin{abstract}
We report a simple homogeneous fluorescence assay for quantification of DNA polymerase function in high throughput. The fluorescence signal is generated by the DNA polymerase triggering opening of a molecular beacon extension of the template strand. A resulting distance alteration is reported by fluorescence resonance energy transfer between two dyes introduced into the molecular beacon stem. We describe real-time reaction profiling of two model DNA polymerases. We demonstrate kinetic characterization, rapid optimization of reaction conditions, and inhibitor profiling using the presented assay. Furthermore, to supersede purification steps in screening procedures of DNA polymerase mutant libraries, detection of enzymatic activity in bacterial expression lysates is described.
\end{abstract}

Key Words: High throughput screening; FRET; DNA polymerase; directed evolution; inhibitor screening; fluorescent probes.

\section{Introduction}

DNA polymerases catalyze the template-directed DNA synthesis in all living organisms occurring in replication, repair, and recombination (1). This central role has rendered these enzymes to key components of numerous biotechnological applications like polymerase chain reaction (PCR), cDNA synthesis for expression profiling, mutagenesis techniques, and genotyping of single nucleotide polymorphisms (SNPs) $(2,3)$. In addition, DNA polymerases represent important drug targets, particularly in the therapy of antiretroviral diseases like acquired immunodeficiency syndrome (AIDS) (4-6). Today, progress in drug discovery is tightly linked to the massive parallel evaluation of large compound libraries like natural product collections or libraries generated by combinatorial synthesis for inhibitor potency (7). Similarly, to tailor DNA polymerases for artificial reaction conditions used in biotechnological applications, directed 
evolution has emerged as a powerful tool to isolate new or improved enzyme functions from genetically engineered protein libraries (8).

Current demands on assay formats for high-throughput screening are high sensitivity to allow minimal assay volumes, ease of automation, and the avoidance of hazardous components like radioisotopes $(7,8)$. Additionally, online profiling of enzymatic activity is much preferred over endpoint measurements of the generated product, since reaction rates can be deduced from slopes of the obtained reaction graphs. This enhances the assays dynamic range for a given substrate concentration especially if a broad range of activity is present in the screened library and adjustment of reaction time and substrate consumption is difficult to achieve for all variants.

We have developed a homogenous, fluorescent assay format for rapid evaluation of DNA polymerase function that allows real-time monitoring of reaction graphs (9). The assay can be used for inhibitor screening and evaluation, rapid optimization of reaction conditions, assessment of kinetic constants, and for the measurement of DNA polymerase activity in crude lysates of bacterial expression cultures. The design of the setup should allow screening of variant libraries for properties like altered fidelity, stability, and processing of artificial substrates. Employment of crude expression lysates simplifies the screening procedure since laborious purification steps can be omitted.

The assay relies on a molecular beacon equipped with fluorescence resonance energy transfer (FRET)-donor and -acceptor dye within an 8mer stem. This stem bears a 3 -extension that allows binding of a primer and acts as template for the DNA polymerase (Fig. 1).

Proceeding DNA synthesis results in opening of the stem region, separation of the dyes and increase of donor emission. We took advantage of the widely used FAM/TAMRA pair (carboxyfluorescein, $N, N$ '-tetramethylrhodamine) for FRET though other dyes might be used that can be bypassed by the DNA polymerase employed $(\mathbf{1 0}, 11)$. The assay setup allows investigation of a wide variety of DNA polymerases. We tested phage T7 DNA polymerase and its $3^{\prime}-5^{\prime}$-exonuclease deficient mutant Sequenase ${ }^{\mathrm{TM}}$, Human immunodeficiency virus type 1 reverse transcriptase (HIV-1 RT), human DNA polymerase $\beta$, bacterial Bacillus stearothermophilus (Bst) DNA Polymerase I (large fragment) and several archaeal thermostable enzymes (Vent ${ }^{\mathrm{TM}}, 3^{\prime}-5^{\prime}$-exonuclease-deficient Vent $\left(\text { Vent }^{-}\right)^{\mathrm{TM}}$ ), and Thermus thermophilus (Tth) DNA polymerase.

In the following, we describe the evaluation of this assay principle with the Klenow Fragment of E. coli $\mathrm{Pol}$ I $\left(3^{\prime}-5^{\prime}\right.$ e- $\left.\mathrm{xO}^{-}, \mathrm{KF}^{-}\right)$, i.e., the assessment of Michaelis-Menten kinetics, optimum concentration of magnesium ions, and detection of DNA polymerase activity in crude bacterial expression lysates. Additionally, we describe the profiling of inhibitor potency for HIV-1 RT with two well-characterized inhibitors of this target enzyme. 


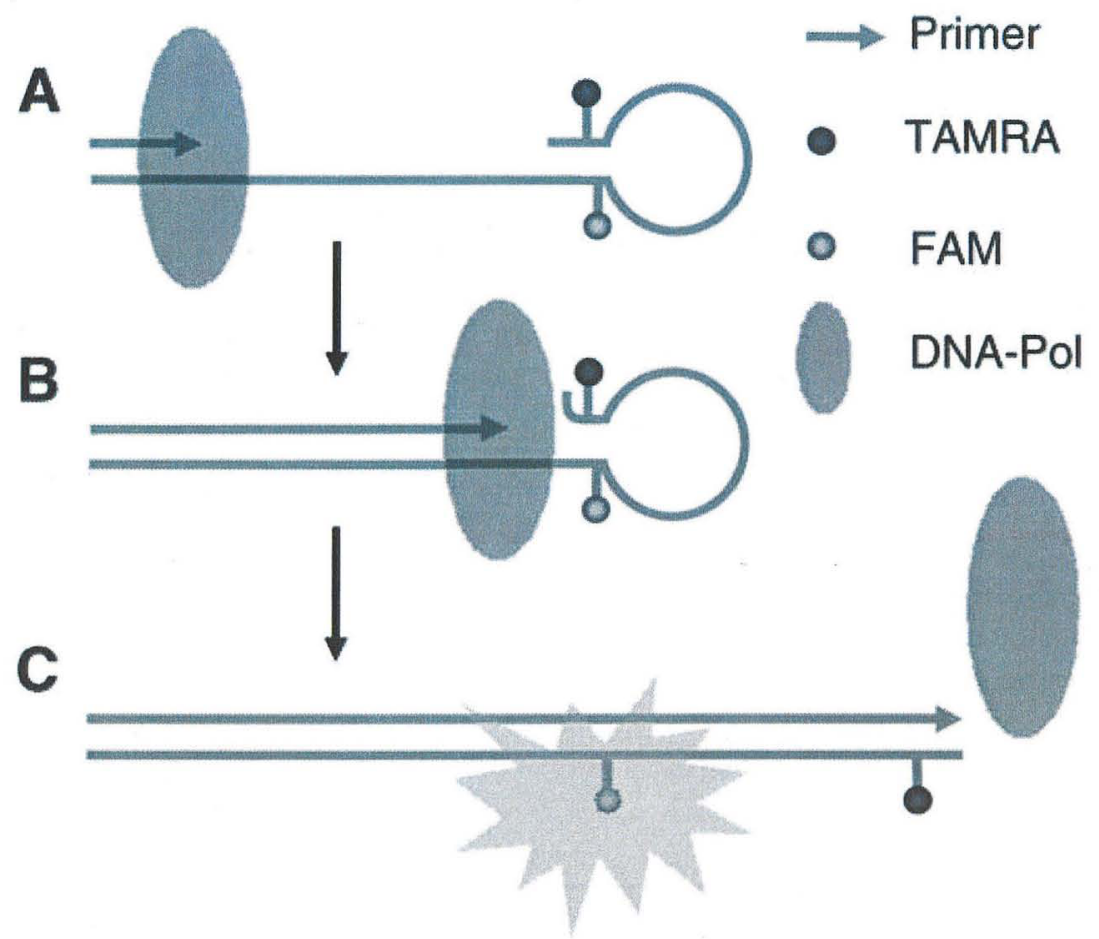

Fig. 1. Assay design for real-time observation of the DNA polymerase reaction. (A) The molecular beacon template labeled with fluorophor (carboxyfluorescein, FAM) and acceptor ( $N, N^{\prime}$-tetramethylrhodamine, TAMRA) bears a loop structure in closed conformation before start of reaction. (B) While extension proceeds, the DNA polymerase opens the stem and prevents reannealing by DNA duplex formation. (C) The increase in the distance between the two labels is reported by restoration of FAM emission. Figure was taken from Ref. (9).

\section{Materials}

\subsection{Design and Synthesis of a Molecular Beacon Template}

The molecular beacon template can be synthesized by the researcher or purchased by a commercial supplier. Several oligonucleotide synthesis companies provide synthesis and purification of long, double internally labeled oligos. These include IBA GmbH (Germany), MWG-Biotech (Germany), Eurogentech (Belgium), and Integrated DNA Technologies (USA).

The construct we used consisted of a 23 mer loop connected by a GC-rich 8 mer stem region and a 43 mer extension with the sequence $5^{\prime}-G G C C C G U^{T A M R A} A G-$ GAG GAA AGG ACA TCT TCT AGC A $U^{F A M} A C G G$ GCC GTC AAG TTC ATGGCC AGT CAA GTC GTC AGA AAT TTC GCA CCA C-3' where italicizes identify the stem sequences. TAMRA and FAM were connected to C5 of 2'-deoxyuridines as 5,6-regioisomeric mixtures. The employed primer had the sequence 5'-(GTG GTG CGA AAT TTC TGA C)-3'. If changing part of the sequence 
is desired, evaluation of the folding properties using David Zukers web-based DNA-folding software mfold is recommended (www.bioinfo.rpi.edu/applications/ $\mathrm{mfold} /$ old/dna/form1.cgi).

Following materials were used for synthesis:

1. ABI 392 DNA/RNA synthesizer (Applied Biosystems, USA).

2. Ultramild phosphoramidites (Glen Research, USA).

3. Fluorescein dT phosphoramidite (cat. no. 10-1056; Glen Research).

4. TAMRA dT phosphoramidite (cat. no. 10-1057; Glen Research).

5. DNA synthesis reagents, controlled pore glass solid supports (1000 ̊ pore size) and deprotection solution of the Ultramild series (Glen Research).

\subsection{Expression of $K F^{-}$}

Expression was achieved using plasmid $\mathrm{pQKlenowExo}{ }^{-}$(kindly provided by Dr. S. Brakmann, Leipzig) (11). The plasmid is derived from pQE30 (Qiagen, Germany) and allows tightly regulated transcription from the T5 promoter by two lac operator sequences.

Following materials were used for expression:

1. Vector $\mathrm{pQKlenowExo}{ }^{-}$(Dr. S. Brakmann).

2. Vector $\mathrm{pQE} 30 \mathrm{Xa}$ (Qiagen).

3. E. coli strains M15 [pREP4] (Qiagen) or XL1blue (Stratagene, USA) (see Note 1).

4. Luria Bertani medium (LB).

5. Super broth.

6. Ampicillin (Sigma, USA).

7. Kanamycin (Sigma).

8. Isopropyl- $\beta$-D-thiogalactopyranoside, $50 \mathrm{mM}$ stock solution (IPTG; Sigma).

9. $1.2 \mathrm{~mL}$ 96-deepwell plates (cat. no. 0030 127.544; Eppendorf, Germany).

10. $2.2 \mathrm{~mL}$ 96-deepwell plates (cat. no. 0030 127.560; Eppendorf).

11. Airpore tape sheets (cat. no. 19571; Qiagen).

12. Lysis buffer: $50 \mathrm{mM} \mathrm{NaH} \mathrm{PO}_{4}, 300 \mathrm{mM} \mathrm{NaCl}(\mathrm{pH} 8.0$ ) containing $1.5 \mathrm{mg} / \mathrm{mL}$ lysozyme (Sigma).

\subsection{Automated Online Profiling of DNA Polymerase Activity by FRET}

1. Fluorescence plate reader with at least one dispenser, shaking option, and filters for. fluorescein excitation and emission like Fluostar, Polarstar (BMG Labtechnologies, Germany) or Fluoroskan Ascent (Thermolabsystems, USA).

2. Black 96 -well fluorescence assay plates, low protein binding (cat. No. 3694, Corning, USA) (see Note 2).

3. Klenow fragment $\left(3^{\prime}-5^{\prime}\right.$-exonuclease-deficient, NEB, USA).

4. HIV-1 reverse transcriptase (Roche, Germany).

5. KF- reaction buffer: $50 \mathrm{mM}$ Tris- $\mathrm{HCl}(\mathrm{pH} 7.3)$ and $1 \mathrm{~m} M$ dithiothreitol (DTT).

6. HIV-1 RT reaction buffer: $50 \mathrm{~m} M$ Tris- $\mathrm{HCl}(\mathrm{pH} 8.3$ ) and $50 \mathrm{mM} \mathrm{KCl}$.

7. $3^{\prime}$-Azido-2'3'-dideoxythymidine-5'-O-triphosphate (AZT TP, IBA GmbH, Germany). 
8. Nevirapine (AIDS Research and Reference Reagent Program, NIAID, NIH).

9. Deoxynucleoside triphosphates (Sigma).

10. $0.1 \mathrm{M} \mathrm{MgCl}_{2}$.

\section{Methods}

\subsection{Synthesis of a Molecular Beacon Template}

In principle, double labeling can be done postsynthetically via aminolinkers or sulfhydryl functions, by direct coupling of labeled phosphoramidites or both (mixed synthesis). If possible, direct coupling of both dyes is recommended in terms of coupling efficiencies and in particular to obtain clean 1:1 ratios of fluorophor and dye. We experienced strong variations in signal-to-noise ratio when DNA was prepared by mixed synthesis. In our case, FAM dT and TAMRA dT phophoramidites were used and Ultramild chemistry was applied since TAMRA is labile under standard deprotection conditions. Synthesis should be performed using $1 \mu \mathrm{mol}$ solid support with 1000 A pore size using standard DNA-coupling protocols, DMT off mode and increased coupling time of $10 \mathrm{~min}$ for the labeled phosphoramidites. Purification by $12 \%$ denaturing PAGE ( $8 M$ urea) using a $1.5 \mathrm{~mm}$ gel and a $50 \mathrm{~cm}$ chamber is strongly recommended to get optimal results (see Note 3). DNA can be stored in $\mathrm{ddH}_{2} \mathrm{O}$ at $-20^{\circ} \mathrm{C}$ protected from light.

\subsection{Expression of $\mathrm{KF}^{-}$}

The following protocol is optimized for automated parallel expression in 96-well plates using a liquid handling station like the Microlab Star (Hamilton Robotics, Switzerland) or the Genesis (Tecan, Switzerland). In principle, all steps except for centrifugation steps can be automated.

1. A volume of $500 \mu \mathrm{L} \mathrm{LB}$ medium containing $100 \mu \mathrm{g} / \mathrm{mL}$ ampicillin (for XL1blue and additionally $25 \mu \mathrm{g} / \mathrm{mL}$ kanamycin when M15 [pREP4] is used) in a well of a $1.2 \mathrm{~mL}$ 96-deepwell plate were inoculated with a single colony of the respective

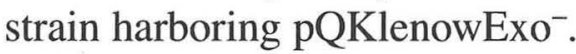

2. Plates were sealed with airpore tape sheets and grown overnight at $37^{\circ} \mathrm{C}$ and 200 rpm shaking (see Note 4).

3. Fifty microliters of overnight cultures were added to $930 \mu \mathrm{L}$ super broth in $2.2 \mathrm{~mL}$ 96-deepwell plates.

4. Cultures were sealed with airpore tape sheets, grown at $37^{\circ} \mathrm{C}$ to an OD600 of $0.6-0.7$ under shaking at $200 \mathrm{rpm}$ and induced by addition of $20 \mu \mathrm{L}$ IPTG $(50 \mathrm{mM}$ resulting in $1 \mathrm{~m} M$ final concentration).

5. After $4 \mathrm{~h}$ shaking at $200 \mathrm{rpm}$ at $37^{\circ} \mathrm{C}$ cells were harvested by centrifugation.

6. For lysis, pellets were subjected to one freeze-thawing step, resuspended in $500 \mu \mathrm{L}$ lysis buffer and shaked for $45 \mathrm{~min}$ at $4^{\circ} \mathrm{C}$.

7. Lysates were cleared by centrifugation and directly used for primer extension reactions. 
8. Control lysates of same strain harboring vector $\mathrm{pQE} 30 \mathrm{Xa}$ without $\mathrm{KF}^{-}$coding insert were prepared identically.

\subsection{Automated Online Profiling of DNA Polymerase Activity by FRET}

\subsubsection{General Procedure for Fluorescent Primer Extension Reactions with a Molecular Beacon Template}

1. Fifty microliter reactions contained $200 \mathrm{n} M$ molecular beacon template, $300 \mathrm{n} M$ primer $10 \mathrm{mM} \mathrm{MgCl}$, and varying amounts of equimolar dATP, dGTP, dCTP, and dTTP in reaction buffer specific for the respective DNA polymerase.

2. Molecular beacon template, primer and $5 \mu \mathrm{L} 10 \times$ reaction buffer were mixed in $40 \mu \mathrm{L}$ total volume/well.

3. Mixture was heated to $95^{\circ} \mathrm{C}$ for $5 \mathrm{~min}$ and was put on room temperature for $1 \mathrm{~h}$.

4. $\mathrm{dNTP}$ and enzyme were added to a total volume of $45 \mu \mathrm{L}$.

5. Mixture was transferred to 96-well assay plate.

6. Plate was preincubated in fluorescence plate reader at $37^{\circ} \mathrm{C}$ for $5 \mathrm{~min}$.

7. Reaction was initiated by automated dispensing of $0.1 \mathrm{M} \mathrm{MgCl}_{2}$ solution followed by a short shaking step ( $3 \mathrm{~s}, 2-3 \mathrm{~mm}$ shaking diameter, $150 \mathrm{rpm}$ ).

8. FAM fluorescence increase was measured in appropriate time intervals by exciting at $485 \mathrm{~nm}$ and emission at $518 \mathrm{~nm}$ as arbitrary units.

\subsubsection{Kinetic Measurements with $\mathrm{KF}^{-}$}

The kinetics of enzymatic DNA synthesis exhibit Michaelis-Menten dependence of substrate concentration, thus an apparent Michaelis-Menten constant $\left(K_{\mathrm{M}}\right)$ can be derived from experiments conducted with a given amount of enzyme and varied substrate concentrations (12).

Reactions were conducted using $1.4 \mathrm{U} \mathrm{KF}^{-}$and a range of $1.25-37.5 \mu \mathrm{M}$ of each dNTP. A measurement of an equivalent reaction mix without $\mathrm{KF}^{-}$was conducted in parallel and subtracted from the data of the experiments including $\mathrm{KF}^{-}$(see Note 6). Reaction graphs were obtained by fluorescence measurements in time intervals of $20 \mathrm{~s}$ for $6.5 \mathrm{~min}$ (Fig. 2a).

Initial apparent reaction rates $\left(v_{\mathrm{i}}\left(F_{518} / \mathrm{min}^{-1}\right)\right)$ were obtained from data by determination of the slopes of the initial linear portion of individual reactions graphs and plotted versus dNTP concentration (Fig. 2b). A $K_{\mathrm{M}}$-value was obtained by nonlinear curve fitting using the Michaelis-Menten equation. We obtained a $K_{\mathrm{M}}$ value $(3.6 \pm 0.2 \mu M)$ that compares well with that measured independently using a conventional radiometric assay $(3.9 \pm 1.1 \mu M)(9)$.

\subsubsection{Rapid Assessment of Optimum Magnesium Ion Concentration}

It is often desirable to rapidly determine optimum conditions of a given enzyme or variant. We employed the assay setup to determine the optimal magnesium chloride concentration in terms of activity. Reactions were performed using 1.4 $\mathrm{U} \mathrm{KF}^{-}$and different $\mathrm{MgCl}_{2}$ concentrations as depicted in Fig. 3 . 

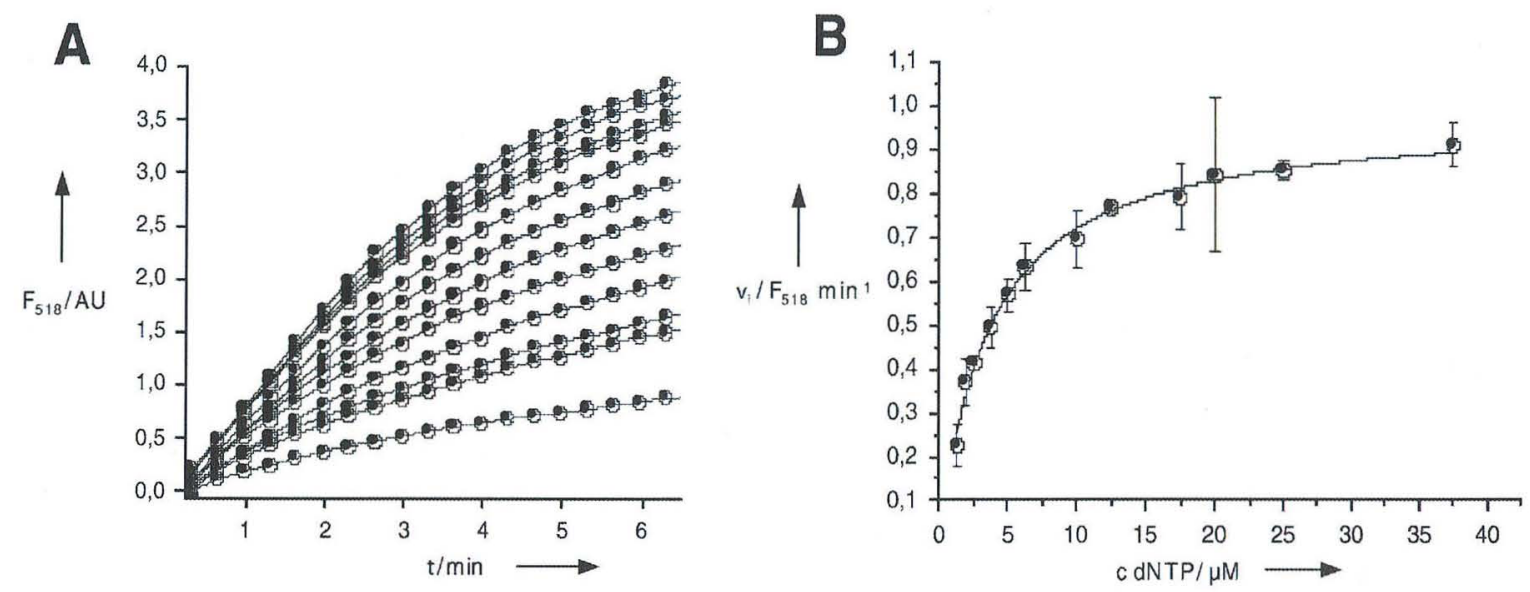

Fig. 2. Fluorescent real-time determination of the $K_{\mathrm{M}}$ constant using $\mathrm{KF}^{-}$. (A) Time courses of reactions employing different dNTP concentrations. Reactions contained $1.4 U \mathrm{KF}^{-}$and dNTP concentrations ranging from $1.25 \mu M$ (lowest curve) to $37.5 \mu M$ (highest curve). Data are results of multiple experiments. For clarity, error bars are not shown. (B) Michaelis-Menten plot of reaction velocities obtained from Fig. 2A. Initial apparent reaction rates $\left(v_{\mathrm{i}}\left(F_{518} / \mathrm{min}^{-1}\right)\right)$ were obtained from data by determination of the slopes of the initial linear portion of individual reaction graphs and plotted versus dNTP concentration. Line graph corresponds to nonlinear curve fit using the Michaelis-Menten equation. Figure was taken from Ref. (9).

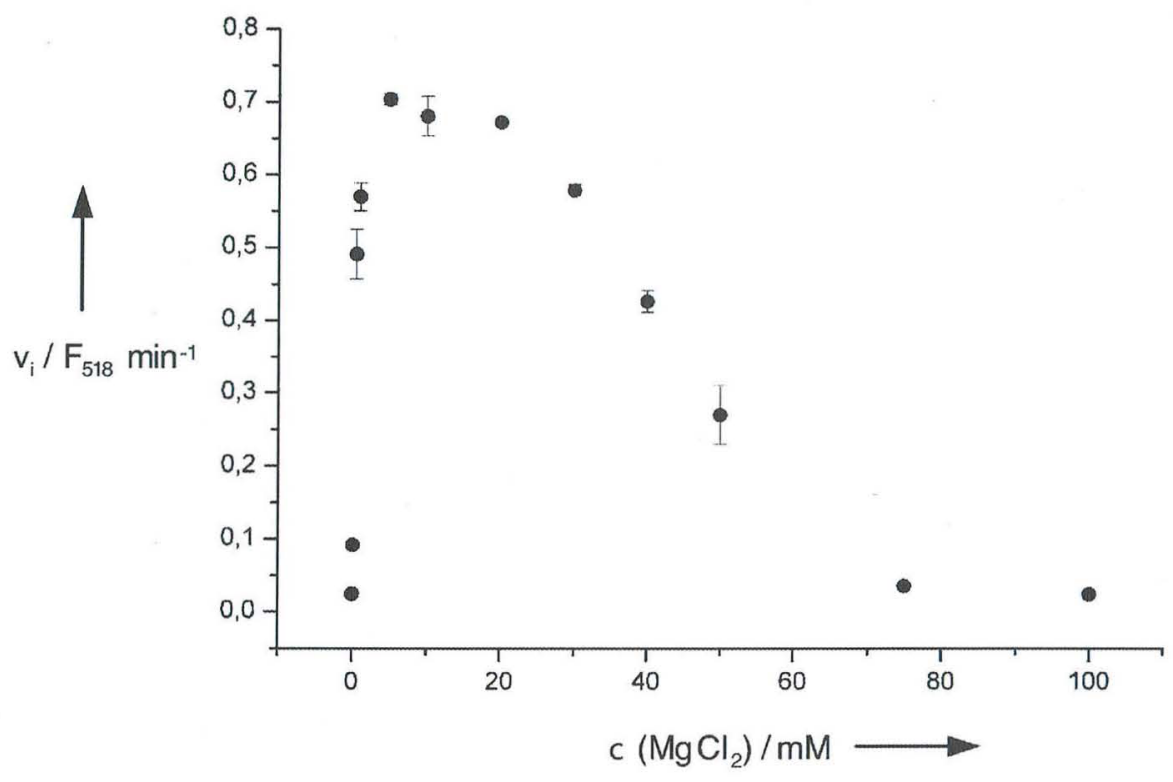

Fig. 3. Rapid assessment of optimal magnesium chloride concentration for $\mathrm{KF}^{-}$ using the molecular beacon template. Reactions contained $1.4 \mathrm{U} \mathrm{KF}^{-}$and different $\mathrm{MgCl}_{2}$ concentrations as depicted. Reactions were started by addition of dNTP to a final concentration of $12.5 \mu M$ of each. Initial apparent reaction rates $\left(v_{\mathrm{i}}\left(F_{518} / \mathrm{min}^{-1}\right)\right)$ were obtained from data by determination of the slopes of the initial linear portion of individual reaction graphs and plotted against dNTP concentration. 
$\mathrm{MgCl}_{2}$ was included during the initial denaturation/renaturation step and reactions were started with $5 \mu \mathrm{L}$ dNTP resulting in $12.5 \mu M$ final concentration. Correction of data and determination of reaction rates $\left(v_{\mathrm{i}}\left(F_{518} / \mathrm{min}^{-1}\right)\right)$ was conducted as in Section 3.3.2 (see Note 6). The obtained optimum concentration of about $10 \mathrm{mM}$ corresponds to the standard $\mathrm{MgCl}_{2}$ concentration used in the literature for $\mathrm{KF}^{-}$.

\subsubsection{Profiling of HIV-1 Reverse Transcriptase Inhibitors}

We used the assay for detection and quantification of interactions of DNA polymerases with inhibitors. We employed HIV-1 RT as model system. In current HIV drug therapy, two classes of RT inhibitors are in use that differ in their mode of action. Non-nucleoside RT inhibitors (NNRTIs) allosterically inhibit RT function, whereas nucleoside RT inhibitors (NRTIs) are first transformed to $5^{\prime}$ - $O$-triphosphates by cellular processes and then incorporated into the nascent DNA strand by the RT to cause chain termination (4-6). To validate whether the assay format rapidly identifies inhibitors of HIV-1 RT acting by both mechanisms, we studied nevirapine and AZT TP representing well characterized NNRTIs and NRTIs, respectively. Reactions were performed using 2.1 U HIV-1 RT and inhibitor in different concentrations. Inhibitors were added to reactions along with $50 \mu M$ dNTP (final concentration) and enzyme before preincubation in the plate reader and reactions were started with $\mathrm{MgCl}_{2}$. Correction of data and determination of reaction rates $\left(v_{\mathrm{i}}\left(F_{518} / \mathrm{min}^{-1}\right)\right)$ was conducted as in Section 3.3.2 (see Note 6). Inhibition profiles from which $\mathrm{IC}_{50}$-values can be deduced were obtained by plotting reaction rates against inhibitor concentration (Fig. 4).

The resulting $\mathrm{IC}_{50}$ values of nevirapine $(0.13 \pm 0.03 \mu M)$ and AZT TP $(2.10 \pm 0.22 \mu M)$ correspond well with reported data indicating the suitability of the assay for faithful inhibitor characterization $(13,14)$.

\subsubsection{Profiling of $\mathrm{KF}^{-}$Activity in Crude Bacterial Lysates}

Fluorescent reactions were conducted under the same conditions as used with purified $\mathrm{KF}^{-} . \mathrm{MgCl}_{2}$ was added before the denaturation/renaturation step and reactions were started with dNTP (200 $M$ final concentration). Instead of purified enzyme, $1 \mu \mathrm{L}$ of cleared lysate of $1 \mathrm{~mL}$ culture was employed (see Section 3.2). As control, same amount of lysate of the control culture (harboring $\mathrm{pQE} 30 \mathrm{Xa}$ ) was used. Data were not corrected as in previously described measurements (see Note 6). The obtained reaction graphs indicate activity only when $\mathrm{KF}^{-}$expressing cultures were employed and exhibit no background activity that might be caused by host factors like DNA polymerases or nucleases (Fig. 5). 


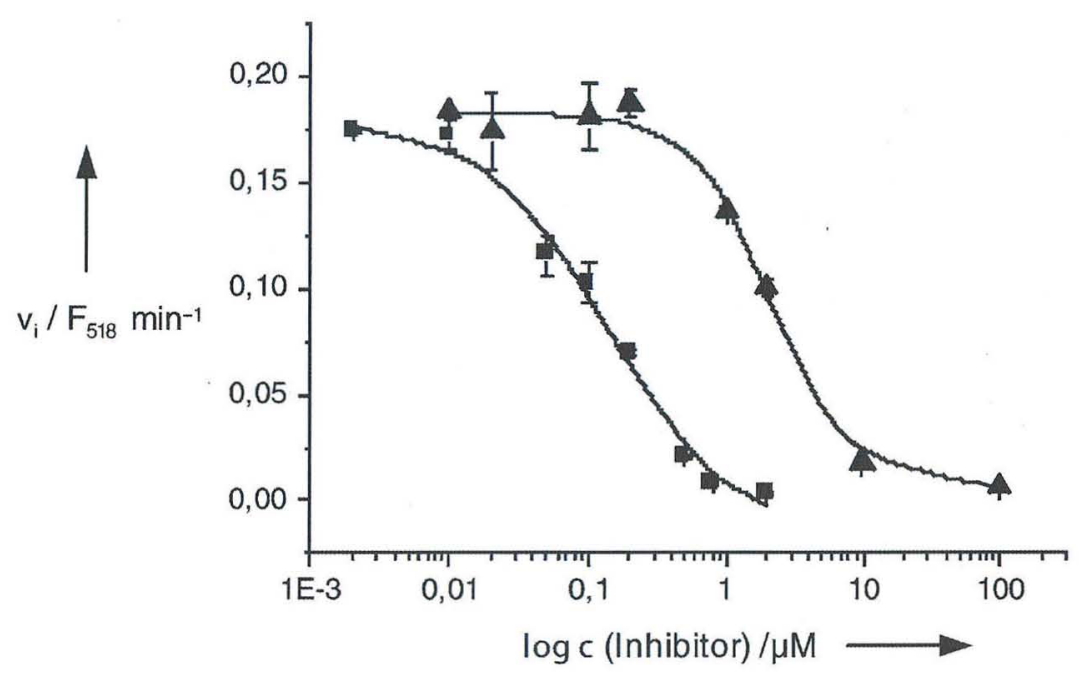

Fig. 4. HIV-1 RT inhibition profiles obtained from measurements with the molecular beacon template. Reactions were conducted using $2.1 \mathrm{U}$ HIV-1 RT and $50 \mu M$ of each dNTP in the presence of inhibitors at different concentrations as depicted. (Triangles) AZT TP and (squares) nevirapine. Initial apparent reaction rates $\left(v_{\mathrm{i}}\right)$ were obtained from data by determination of the slopes of the initial linear portion of individual reaction graphs. Figure was taken from Ref. (9).

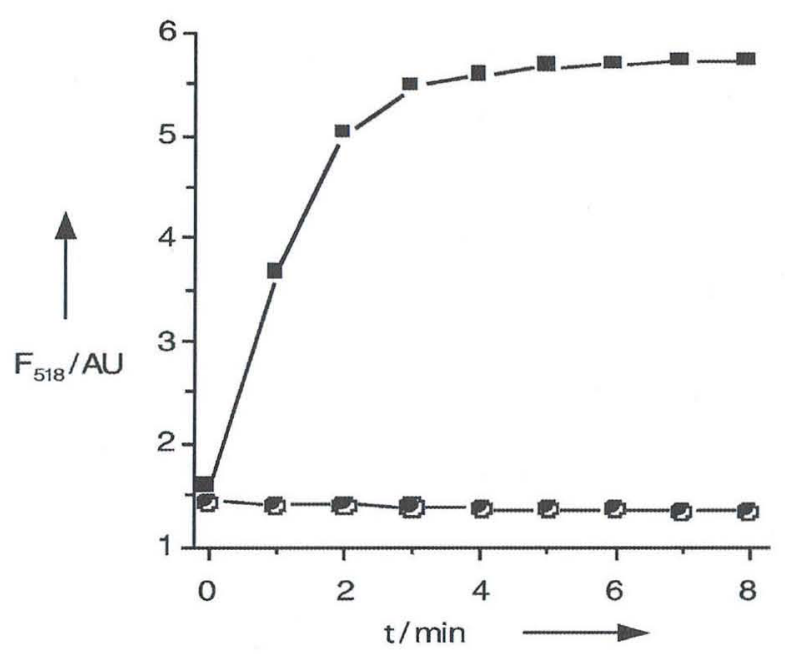

Fig. 5. Primer extension reactions promoted by crude lysates of $\mathrm{KF}^{-}$overexpressing E. coli cultures (strain M15 [pREP4]) using the molecular beacon template. Reactions contained $10 \mathrm{mM} \mathrm{MgCl}$ and lysate of expression-induced M15 [pREP4] cultures and were started by addition of dNTP to a final concentration of $200 \mu M$. (Squares) Reactions employing lysate of M15 [pREP4] cells harboring the $\mathrm{KF}^{-}$coding vector $\mathrm{pQK}$ lenowExo $4 \mathrm{~h}$ after induction of expression. (Circles) Reactions employing lysate of M15 [pREP4] cells harboring the noncoding vector pQE30Xa $4 \mathrm{~h}$ after induction of expression. Data are results of multiple experiments. Presented graphs correspond to raw data without correction (see Note 6). Figure was taken from Ref. (9). 


\section{Notes}

1. For tight control, a high level of lac repressor protein is needed. This can be achieved by cotransformation with pREP4 (Qiagen) that encodes lac repressor or by usage of strains carrying the lacI ${ }^{\mathrm{q}}$ genotype (like XL1blue) that increases lac repressor concentration about 10 -fold.

2. Plates have unusual small wells and protocols are optimized for these plates. The described $50 \mu \mathrm{L}$ reaction volumes are not applicable to regular 96-well plates.

3. Gel temperature should be constantly at $45-50^{\circ} \mathrm{C}$ to avoid formation of secondary structures by the GC-rich stem. If the crush and soak method is used for gel extraction, sample should be incubated at $-80^{\circ} \mathrm{C}$ thoroughly for precipitation. Solubility of the oligonucleotide in $70 \%$ ethanol is enhanced by the dyes and a second precipitation step might be necessary.

4. Shaking diameter seemed not to be critical. We shaked with a diameter of several centimeters with good expression yield so that a common incubator can be used.

5. For generation of mutant libraries, long-term storage of the lysates is desirable. This can be done by addition of three volumes storage buffer $(50 \mathrm{~m} M$ Tris- $\mathrm{HCl}$ (pH 7.3), $1 \mathrm{~m} M$ DTT, $0.1 \mathrm{~m} M$ EDTA, $1 \mathrm{~m} M$ PMSF, $1 \mathrm{~m} M$ benzamidine, $1 \mu \mathrm{g} / \mathrm{mL}$ leupeptine, and $1 \mu \mathrm{g} / \mathrm{mL}$ aprotinine) after lysis. Expression culture and lysis volume should be scaled by a factor of 0.6 in this case to allow proper handling in 96-deepwell plates. Samples can be stored at $-80^{\circ} \mathrm{C}$ and tolerate at least three freeze-thawing steps without detectable loss in activity.

6. We observed slight photobleaching of FAM that can be corrected by this procedure. This effect can be observed in the negative control of Fig. 5 that is not corrected. In case of the optimization experiment for $\mathrm{MgCl}_{2}$, a quenching effect is observable and initial fluorescence decreases with increasing magnesium concentration. This effect is eliminated by analyzing reaction rates as described rather than absolute fluorescence data.

\section{Acknowledgments}

The author thank Prof. Dr. Andreas Marx at the University of Konstanz for continuous support and helpful discussions.

\section{References}

1. Kornberg, D. G. and Baker, T. A. (1991) DNA Replication. W. H. Freeman, New York, NY.

2. Sambrook, J. and Russell, D. W. (2001) Molecular cloning: A Laboratory Manual, Cold Spring Harbor Laboratory Press, Cold Spring Harbor, NY.

3. Shi, M. M. (2001) Technologies for individual genotyping: detection of genetic polymorphisms in drug targets and disease genes. Clin. Chem. 47, 164-172.

4. Naeger, L. K. and Miller, M. D. (2001) Mechanisms of HIV-1 nucleoside reverse transcriptase inhibitor resistance: is it all figured out? Curr. Opin. Invest. Drugs 2 , 335-339.

5. Tozser, J. T. (2001) HIV inhibitors: problems and reality. Ann. N. Y. Acad. Sci. 946, $145-159$. 
6. Pillay, D., Taylor, S., and Richman, D. D. (2000) Incidence and impact of resistance against approved antiretroviral drugs. Rev. Med. Virol. 10, 231-253.

7. Hertzberg, R. P. and Pope, A. J. (2000) High-throughput screening: new technology for the 21st century. Curr. Opin. Chem. Biol. 4, 445-451.

8. Olsen, M., Iverson, B., and Georgiou, G. (2000) High-throughput screening of enzyme libraries. Curr. Opin. Biotechnol. 11, 331-337.

9. Summerer, D. and Marx, A. (2002) A molecular beacon for quantitative monitoring of the DNA polymerase reaction in real time. Angew. Chem. Int. Ed. 41 (19), 3620-3622.

10. Brakmann, S. and Lobermann, S. (2001) High-Density Labeling of DNA: Preparation and characterization of the target material for single-molecule sequencing. Angew. Chem. Int. Ed. 40, 1427-1429.

11. Brakmann, S. and Nieckchen, P. (2001) The large fragment of Escherichia coli DNA polymerase I can synthesize DNA exclusively from fluorescently labeled nucleotides. Chem. Bio. Chem 2, 773-777.

12. Fersht, A. (2000) Structure and Mechanism in Protein Science, Freeman, New York, NY.

13. Reardon, J. E. and Miller, W. H. (1990) Human immunodeficiency virus reverse transcriptase. Substrate and inhibitor kinetics with thymidine $5^{\prime}$-triphosphate and 3'-azido-3'-deoxythymidine 5'-triphosphate. J. Biol. Chem. 265, 20,302-20,307.

14. Merluzzi, V. J., Hargrave, K. D., Labadia, M., et al. (1990) Inhibition of HIV-1 replication by a nonnucleoside reverse transcriptase inhibitor. Science, 250, 1411-1413. 